\title{
Magnetic Nanocomposite Cilia Energy Harvester
}

\author{
Mohammed Asadullah Khan, Ahmed Alfadhel, and Jürgen Kosel \\ King Abdullah University of Science and Technology (KAUST), Computer, Electrical and Mathematical Sciences and \\ Engineering Division (CEMSE), Thuwal, Kingdom of Saudi Arabia
}

\begin{abstract}
An energy harvester capable of converting low frequency vibrations into electrical energy is presented. The operating principle, fabrication process and output characteristics at different frequencies are discussed. The harvester is realized by fabricating an array of polydimethylsiloxane (PDMS) - iron nanowire nanocomposite cilia on a planar coil array. Each coil element consists of 14 turns and occupies an area of $600 \mu \mathrm{m} \times 600 \mu \mathrm{m}$. The cilia are arranged in a 12x5 array and each cilium is $250 \mu \mathrm{m}$ wide and $2 \mathrm{~mm}$ long. The magnetic characteristics of the fabricated cilia indicate that the nanowires are well aligned inside of the nanocomposite, increasing the efficiency of energy harvesting. The energy harvester occupies an area of $66.96 \mathrm{~mm}^{2}$ and produces an output r.m.s voltage of $206.47 \mu \mathrm{V}$, when excited by a $40 \mathrm{~Hz}$ vibration of $1 \mathrm{~mm}$ amplitude.
\end{abstract}

\section{Index Terms-Cilia, Energy Harvester, Magnetic Nanowire, Nanocomposite.}

\section{INTRODUCTION}

$\mathrm{M}_{\mathrm{b}}^{\mathrm{n}}$ INIATURIZED devices have seen a phenomenal growth in both performance and market penetration thanks to the technological progress that is following Moore's Law [1]. This has ushered in a new paradigm of ubiquitous connectivity with exciting applications in the fields of point of care diagnostics, multimedia, wearable computing, environmental sensing and urban planning, to name a few. Since these devices are required to operate in a self-sufficient manner, powering them is a significant challenge.

Conventionally, electrochemical batteries are used to power such devices, but they require frequent recharging and eventual replacement, operations which might be extremely difficult and prohibitively expensive. Ambient energy is often found in the sensing environment in the form of light, heat, vibrations, etc. Mechanical vibrations in particular are ubiquitous in most sensing environments from sources such as human movement, air conditioning units, refrigeration units, vehicle vibrations, sound, etc. These vibrations can be harnessed to power miniature sensing and computing systems to enable a robust, self-sufficient and truly autonomous electronic ecosystem [2]. Mechanical energy harvesters utilize phenomena such as electromagnetism, piezoelectricity, triboelectricity, etc. to convert mechanical vibrations to electrical energy [3].

Electromagnetic harvesters are quite popular in meso and macro scale systems owing to ease of design and high reliability [4]. However, due to difficulty in fabricating the coils and incorporating a micro-scale permanent magnet, the

Manuscript received April 1, 2015; revised May 15, 2015 and June 1, 2015; accepted July 1, 2015. Date of publication July 10, 2015; date of current version July 31, 2015. (Dates will be inserted by IEEE; "published"

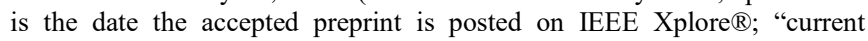
version" is the date the typeset version is posted on Xplore ()). Corresponding author: Jurgen Kosel (e-mail: jurgen.kosel@kaust.edu.sa)." IEEE TRANSACTIONS ON MAGNETICS discourages courtesy authorship; please use the Acknowledgment section to thank your colleagues for routine contributions.

Color versions of one or more of the figures in this paper are available online at http://ieeexplore.iee.org.

Digital Object Identifier (inserted by IEEE). miniaturization of electromagnetic harvesters has proven to be quite challenging. In most micro-scale electromagnetic energy harvesters, a discrete permanent magnet, affixed after the microfabrication process serves as the flux source [5],[6]. This solution is hardly elegant and does not lend itself to easy batch manufacturing. In some designs even the coil is a conventional wire wound coil [7], which cannot be miniaturized and is not compatible with CMOS fabrication flow.

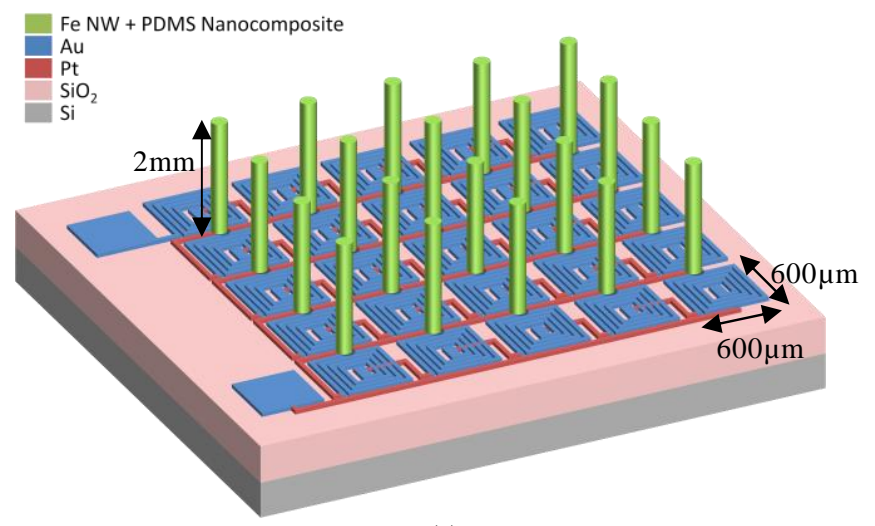

(a)

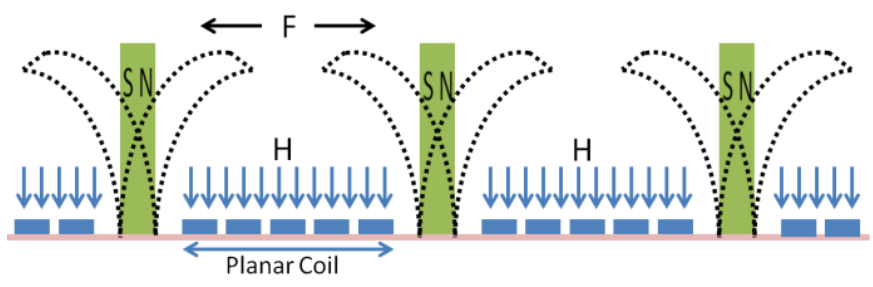

(b)

Fig. 1. (a) Schematic of magnetic nanocomposite cilia energy harvester (b) Illustration of harvester operation, indicating the movement of cilia with applied force $\mathrm{F}$ and magnetic field component perpendicular to coil array, $\mathrm{H}$.

In this work, we provide a solution to the permanent magnet integration issue by using a permanent magnetic nanocomposite instead of a discrete permanent magnet. The composite fulfils the need for a low rigidity material with remanent magnetization. Further, it can be easily processed by spin coating, drop casting and molding [8]-[10]. For this application, the composite is molded to form bio-inspired hair like structures known as cilia. 


\section{WORKING PRINCIPLE}

The energy harvester schematic is shown in fig. 1(a). It includes an array of cilia, made using a PDMS - iron nanowire composite. Due to high shape anisotropy, iron nanowires are permanently magnetized along their length and PDMS being a soft polymer has a very low stiffness. Thus, the high aspect ratio cilia are designed to have high remanent magnetization and low rigidity.

In order to simplify our analysis, we assume that the aligned nanocomposite cilia behave similar to a cylindrical magnetic dipole, magnetized perpendicular to its axis. When an oscillatory force is applied along the magnetization axis, the cilia vibrate as depicted in fig. 1(b). When the force acts along the magnetization direction, the north pole of a cilium comes closer to the substrate, while simultaneously the south pole is driven further away from the substrate. Thus, the magnetic field intensity at the substrate increases. When the movement of neighboring cilia are taken into account, this change is further enhanced. Conversely, when force is applied opposite to the direction of magnetization, the magnetic field intensity at the substrate decreases. Thus, when the cilia array is vibrated along the magnetization direction, an oscillating magnetic field is set up along the substrate.

This oscillating magnetic field is converted into electrical potential using planar coils fabricated on the substrate. From Faraday's Law of Induction, the electric potential, $V(t)$ generated by a single turn of wire enclosing an area, $A$, when subject to a magnetic field $H(t)$ is given as

$$
V(t)=-\frac{d \phi(t)}{d t}=-\mu_{0} \cdot A \cdot \frac{d}{d t}(H(t)),
$$

where $\mu_{o}$ is the magnetic permeability of free space. If the cilia array is stimulated by a monotone mechanical vibration of frequency $f$, the resultant magnetic field will also have a frequency $f$. Applying this condition to equation (1), the amplitude of the voltage generated by a single $\mathrm{N}$-turn planar coil is given as

$$
V_{m}=2 . \pi \cdot f \cdot \mu_{0} \cdot \sum_{k=1}^{N}\left(H_{m k} \cdot A_{k}\right)
$$

where $A_{k}$ is the area of the $\mathrm{k}^{\text {th }}$ turn and $H_{m k}$ is the average amplitude of the magnetic field associated with the $\mathrm{k}^{\text {th }}$ turn of the coil. Since the coils are connected in series, the voltages can be summed up to obtain the voltage generated by the array.

\section{FABRICATION}

The energy harvester fabrication flow consists of the following processes: A. Fe Nanowire Growth B. Coil array fabrication and C. Nanocomposite preparation and cilia molding. These processes are explained in detail subsequently.

\section{A. Fe Nanowire Growth}

Iron nanowires are fabricated by electrodeposition into aluminum oxide templates [11]. We start with a pure $\mathrm{Al}$ disk, whose surface is cleaned by electropolishing. The disk is subject to two oxidation steps (in an oxalic acid solution at - $1.6^{\circ} \mathrm{C}$ ), which results in an alumina membrane with uniform pore distribution.

The backside $\mathrm{Al}$ is etched to release the alumina membrane and gold electrode is deposited on the backside. This is followed by electrodeposition in a $\mathrm{FeCl}_{3}$ solution. Gold is then removed from the backside of the disk by dry argon etching. The Fe nanowires are released by etching away the alumina template in $\mathrm{CrO}_{3}$ and phosphoric acid solution. The released wires are thoroughly washed with ethanol to remove any traces of chrome.

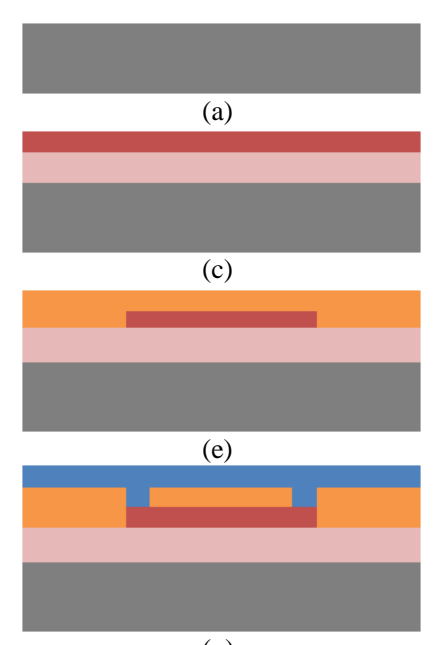

(g)

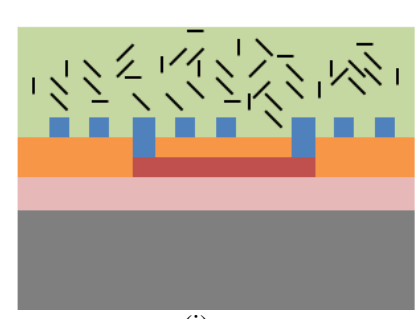

(i)

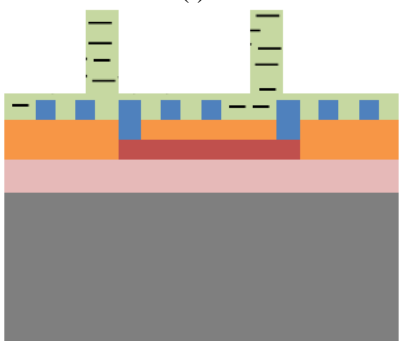

(k)

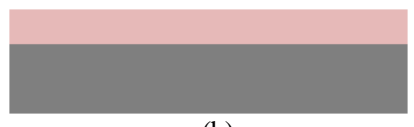

(b)

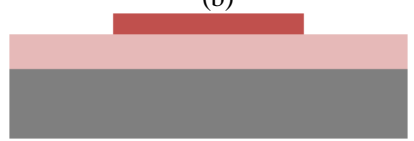

(d)

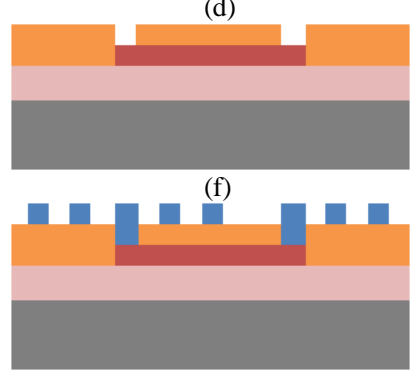

(h)

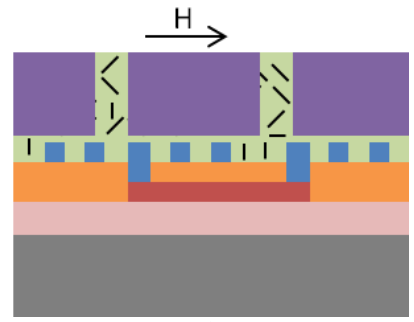

(j)

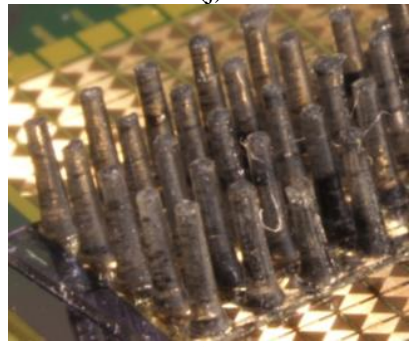

(1) $\square \mathrm{Si} \backsim \mathrm{SiO}_{2} \square \mathrm{Pt} \backsim \mathrm{Si}_{3} \mathrm{~N}_{4} \square \mathrm{Au}=$ Nanocomposite $\square \mathrm{PMMA}$

Fig. 2. Overview of fabrication process (not drawn to scale). (a) $\mathrm{Si}\langle 100\rangle$ wafer, (b) $\mathrm{SiO}_{2}$ thermal growth, (c) Pt deposition by DC sputtering, (d) Litho and Ar dry etch, (e) $\mathrm{Si}_{3} \mathrm{~N}_{4}$ PECVD deposition, (f) Litho and $\mathrm{Si}_{3} \mathrm{~N}_{4}$ etch, (g) Au deposition by DC sputtering, (h) Litho and Au etch, (i) Nanocomposite deposition, (j) Nanocomposite molding, curing and alignment (k) Demolded cilia and (1) Photograph of energy harvester.

\section{B. Coil Array Fabrication}

The coil array is fabricated on a $\langle 100\rangle \mathrm{Si}$ substrate. A $500 \mathrm{~nm}$ thick silicon dioxide is grown on the substrate by a dry-wet-dry cycle to provide electrical isolation. A 3 layer metal-insulator-metal process, detailed below, is used to form the array.

A thin layer of titanium $(10 \mathrm{~nm})$ is deposited by DC 
sputtering in an argon ambient to provide better adhesion. Subsequent to this, without breaking vacuum, a $120 \mathrm{~nm}$ platinum layer is deposited. This layer is then patterned by a standard mask-based photolithography step, followed by a dry argon etch step to form the interconnect layer.

Subsequently, a $150 \mathrm{~nm}$ thick layer of silicon nitride is deposited on the substrate using plasma enhanced chemical vapor deposition. The process is carried out at $300^{\circ} \mathrm{C}$ with silane, ammonia and nitrogen precursors. The plasma frequency is alternated between $300 \mathrm{kHz}$ for $6 \mathrm{~s}$ and 13.56 $\mathrm{MHz}$ for $14 \mathrm{~s}$ to help reduce the stress in the nitride layer. A photolithography step followed by reactive ion etching with trifluoromethane $\left(\mathrm{CHF}_{3}\right)$ and Sulphur hexafluoride $\left(\mathrm{SF}_{6}\right)$ is used to pattern this layer to create vias that connect the interconnect layer with the coil layer.

The next step is deposition of an adhesion layer $(10 \mathrm{~nm}$ titanium), followed by a $1200 \mathrm{~nm}$ thick gold layer using DC sputtering in an argon ambient. This is followed by photolithography and argon ion etch to form the coils.

\section{Nanocomposite preparation and Cilia molding}

Slygard $^{\circledR} 184$ PDMS is prepared by mixing the pre-polymer and the curing agent in a 10:1 weight ratio, as recommended by the manufacturer. Fe nanowires are mixed with PDMS in a $4 \%$ volume ratio by mechanical stirring to form the nanocomposite. The nanocomposite is then subjected to vacuum desiccation to remove microbubbles and traces of ethanol. A $10 \mu \mathrm{m}$ thick layer of pure PDMS is spun coated on the coil array at $1000 \mathrm{rpm}$. This layer prevents accidental shorting of coils by the nanowires in the nanocomposite.

A $2 \mathrm{~mm}$ thick poly (methyl methacrylate) (PMMA) sheet is patterned with $\mathrm{CO}_{2}$ laser cutter (Universal Laser Systems Inc. PLS 6.75) to form a mold consisting of $12 \times 5$ array of $250 \mu \mathrm{m}$ wide holes separated by $1 \mathrm{~mm}$ gaps. The nanocomposite is drop cast on the coil array and then the PMMA mold is mounted on top of it. This structure is then placed in a vacuum desiccator for $15 \mathrm{~min}$, which removes microbubbles and aids in proper filling of the mold pores. Following this, the structure is cured at $90^{\circ} \mathrm{C}$ for an hour, in the presence of a magnetic field perpendicular to the cilia axis to align the nanowires in the composite in one direction. A close-up of the cilia can be seen in fig. 2(1).

\section{RESULTS AND DISCUSSION}

\section{A. Magnetic Characterization}

Magnetization curves for a single cilium are obtained at $297 \mathrm{~K}$ in the transverse and axial direction using vibrating sample magnetometer. The cilium is firmly affixed in the sample holder using silicone gel to prevent any movement. The applied field was varied from $-10 \mathrm{kOe}$ to $10 \mathrm{kOe}$, while oscillating the holder at $83 \mathrm{~Hz}$ with $1 \mathrm{~mm}$ amplitude. The resultant curves, shown in fig. 3 , reveal that the remanent magnetization in the transverse direction is 10.67 times that in the axial direction. Further the coercive field in the transverse direction is $-1.75 \mathrm{kOe}$, while in the axial direction it is only $0.296 \mathrm{kOe}$. These curves imply that the in-situ magnetic alignment procedure followed while curing the nanocomposite is quite effective.

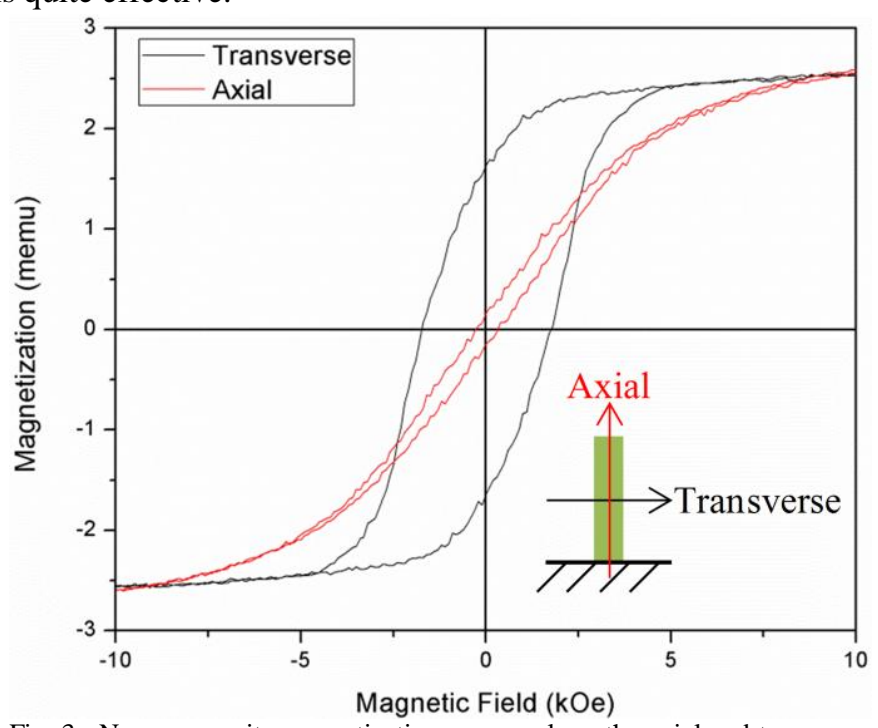

Fig. 3. Nanocomposite magnetization curves, along the axial and transverse directions.

\section{B. Energy Harvesting}

Mechanical vibrations are generated using a TIRA GmbH vibration testing system, which comprises of a shaker, sine vibration controller and power amplifier. A feedback signal generated by the accelerometer mounted on the shaker is used by the controller to generate either constant acceleration or constant displacement vibrations. Unlike conventional energy harvesters that need to be mounted on the vibration source, this harvester functions when the cilia are in contact with the vibration source. In order to facilitate this testing a custom stage with an extended arm is made out of PMMA for the shaker.

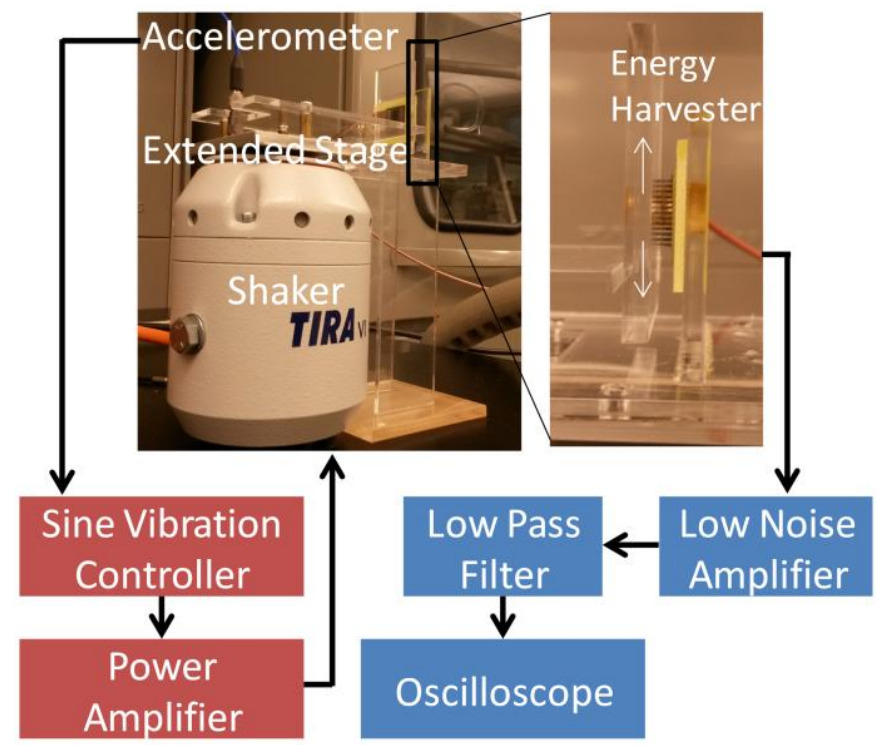

Fig. 4. Block Diagram of the test setup. The zoomed in photograph shows the extended stage of the shaker in contact with the cilia.

The harvester is mounted on a printed circuit board, through which contact is made with a SubMiniature version A (SMA) female connector. The energy harvester is connected to a low 
noise preamplifier (Stanford Research Systems SR-650), which eases detection of the output voltage. The input impedance of the preamplifier $(100 \mathrm{M} \Omega)$ is almost 3 orders of magnitude greater than the internal impedance of the harvester, thus preventing electrical loading. This is followed by a low pass Butterworth filter (Stanford Research Systems SIM965) with a cut-off frequency of $50 \mathrm{~Hz}$ and a $48 \mathrm{~dB} /$ oct. rolloff, which helps eliminate $60 \mathrm{~Hz}$ power line pickup. The filtered output is measured using a digital storage oscilloscope

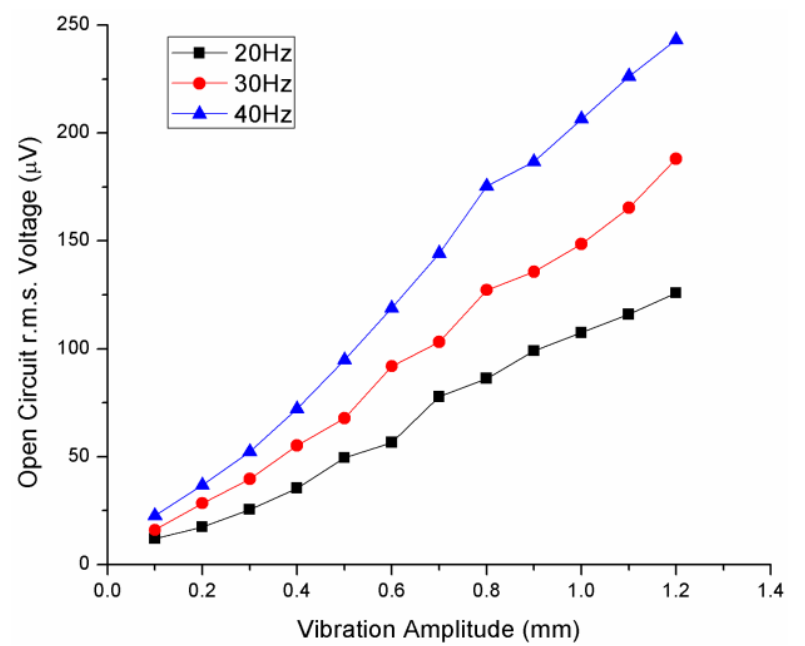

Fig. 5. Open circuit r.m.s. voltage vs. vibration amplitude at $20 \mathrm{~Hz}, 30 \mathrm{~Hz}$ and $40 \mathrm{~Hz}$.

(Agilent Technologies DSO1012A).

The energy harvester is mounted on a custom stand made of PMMA and as shown in fig. 4, it is placed such that the cilia are in contact with the extended stage of the shaker. The shaker is operated in constant displacement mode and the open circuit voltage is measured at $20 \mathrm{~Hz}, 30 \mathrm{~Hz}$ and $40 \mathrm{~Hz}$. The experiment is repeated while varying the vibration amplitude from $0.1 \mathrm{~mm}$ to $1.2 \mathrm{~mm}$ in steps of $0.1 \mathrm{~mm}$. The resultant characteristics are depicted in fig. 5. As the vibration amplitude increases the amplitude of the magnetic field driving the planar coil array also increases, thereby resulting in greater output voltage. As expected from equation (2), a higher voltage is obtained at higher frequencies for the same

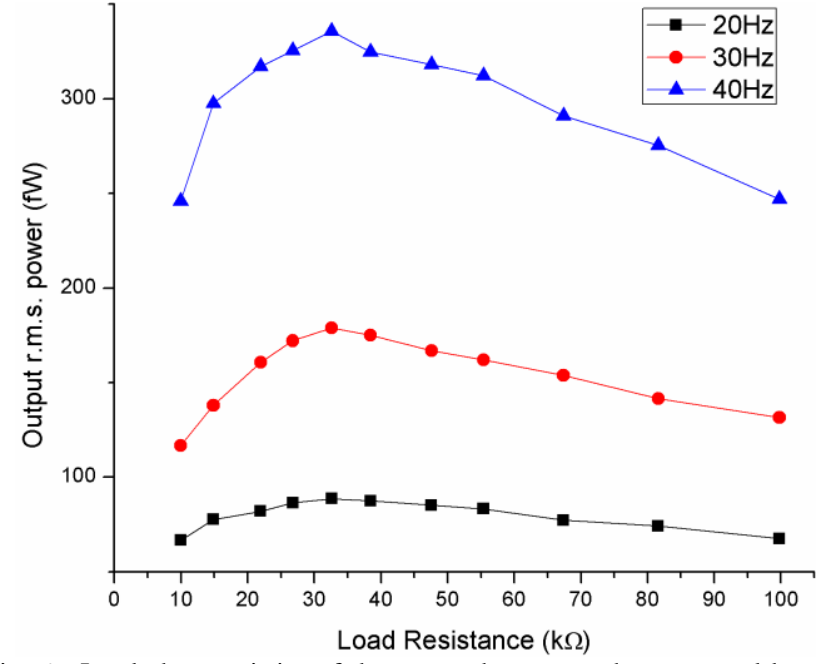

Fig. 6. Load characteristics of the energy harvester when powered by a 1 $\mathrm{mm}$ amplitude vibration. vibration amplitude.

In order to measure the load characteristics of the energy harvester, different resistors between $10 \mathrm{k} \Omega$ and $100 \mathrm{k} \Omega$ are connected across the energy harvester and the resultant output voltage is measured. The R.M.S power is obtained using the expression, $\mathrm{P}_{\mathrm{rms}}=\mathrm{V}_{\mathrm{rms}}^{2} / \mathrm{R}_{\mathrm{L}}$. The experiment is performed at 20 $\mathrm{Hz}, 30 \mathrm{~Hz}$ and $40 \mathrm{~Hz}$ with a vibration amplitude of $1 \mathrm{~mm}$. It can be seen from fig. 6 that maximum power transfer occurs at a load resistance of $33 \mathrm{k} \Omega$. This correlates well with the measured internal impedance of the energy harvester $(32.5 \mathrm{k} \Omega)$.

\section{CONCLUSION}

A magnetic nanocomposite cilia energy harvester capable of scavenging low frequency vibrations is presented in this paper. A permanently magnetized nanocomposite is used as a source of magnetic flux instead of permanent magnets commonly used in conventional harvesters. In-situ magnetic alignment while curing the nanocomposite polymer results in $0.64 \mathrm{M}_{\mathrm{r}} / \mathrm{M}_{\mathrm{s}}$ ratio in the transverse direction and $0.06 \mathrm{M}_{\mathrm{r}} / \mathrm{M}_{\mathrm{s}}$ in the axial direction. This indicates a high degree of $\mathrm{Fe}$ nanowire alignment in the desired direction. The energy harvester output increases with increase in frequency and vibration amplitude. When excited by a $40 \mathrm{~Hz}$ vibration of $1 \mathrm{~mm}$ amplitude with a matched load of $33 \mathrm{k} \Omega$, the energy harvester with a size of only $66.96 \mathrm{~mm}^{2}$ produces $104.7 \mu \mathrm{V}$ r.m.s. voltage and 335.8 fW r.m.s power, which results in $1.98 \mathrm{pW} / \mathrm{cm}^{3}$ power density. The size of the harvester can easily be adjusted to accommodate different applications and variations in the coil and cilia designs can be considered for optimizing the harvesting performance.

\section{REFERENCES}

[1] G. E. Moore, "Progress in digital integrated electronics," 1975 International Electron Devices Meeting, vol.21, pp.11-13, 1975.

[2] S. Roundy, P. K. Wright, and J. Rabaey, "A study of low level vibrations as a power source for wireless sensor nodes," Computer communications, vol. 26, no. 11, pp. 1131-1144, Jul. 2003.

[3] S. P. Beeby, M. J. Tudor, and N. M. White. "Energy harvesting vibration sources for microsystems applications," Measurement science and technology, vol. 17, no. 12, pp. R175-R195, 2006.

[4] D. P. Arnold, "Review of microscale magnetic power generation," IEEE Transactions on Magnetics, vol. 43, no. 11, pp. 3940-3951, Nov. 2007.

[5] P. Wang, K. Tanaka, S. Sugiyama, et al., "A micro electromagnetic low level vibration energy harvester based on MEMS technology," Microsystem Technology, vol. 15, no. 6, pp. 941-951, June 2009.

[6] R. Amirtharajah, and A. P. Chandrakasan. "Self-powered signal processing using vibration-based power generation," IEEE Journal of Solid-State Circuits, vol. 33, no. 5 , pp. 687-695, May 1998.

[7] A. Foisal, C Hong, and GS Chung, "Multi-frequency electromagnetic energy harvester using a magnetic spring cantilever," Sensors and Actuators A: Physical, vol. 182, pp. 106-113, Aug. 2012.

[8] A. Alfadhel, B. Li, A. Zaher, et al., "A magnetic nanocomposite for biomimetic flow sensing," Lab on a Chip, vol. 14, no. 22, pp. 43624369, 2014.

[9] M. Alnassar, A. Alfadhel, Yu. P. Ivanov, et al., "Magnetoelectric Polymer Nanocomposite for Flexible Electronics," Journal Applied Physics, vol. 117, no. 17, pp. 17D711, May 2015.

[10] B. Zhou, W. Xu, A. Syed, et al.," Design and fabrication of magnetically functionalized flexible micropillar arrays for rapid and reversible microfluidic mixing," Lab On a Chip, vol. 15, no. 9, pp. 2125-2132, 2015.

[11] A. Alfadhel and J. Kosel. "Magnetic Nanocomposite Cilia Tactile Sensor", Advanced Materials, 2015. 